\title{
Avaliação dos Efeitos da Radiação Gama nos Teores de Carotenóides, Ácido Ascórbico e Açúcares do Fruto Buriti do Brejo (Mauritia flexuosa L.)
}

Antonio Luís dos Santos LIMA', Keila dos Santos Cople LIMA², Maysa Joppert COELHO³, Jaqueline Michele SILVA $^{4}$, Ronoel Luiz de Oliveira GODOY ${ }^{5}$, Sidney PACHECO ${ }^{6}$

\section{RESUMO}

Buriti do Brejo (Mauritia flexuosa L.) é um típico fruto da Amazônia, Nordeste e Centro-Oeste brasileiro, muito utilizado na culinária para produção de sucos, doces e vinhos. É considerado uma excelente fonte de carotenóides precursores da vitamina A, apresentando maior concentração de $\beta$-caroteno. Além dos carotenóides também encontramos ácido ascórbico e açúcares. $\mathrm{O}$ ácido ascórbico administrado em quantidades suficientes pode prevenir os sinais clínicos de deficiência conhecida como escorbuto. O emprego da radiação ionizante gama tem mostrado um potencial efeito na redução de perdas pós-colheita, mantendo a qualidade nutricional dos alimentos. O presente trabalho teve como objetivo avaliar os efeitos da radiação gama, nas doses de 0,5 kGy e 1,0 kGy, na concentração de carotenóides totais, ácido ascórbico e açúcares do buriti. A determinação dos carotenos ( $\alpha, \beta$ e luteína), ácido ascórbico e açúcares foi realizada por cromatografia líquida de alta eficiência (CLAE) e os carotenóides totais por espectrofotometria. Os resultados demonstraram que o Buriti é uma excelente fonte de carotenóides totais $(44600 \mu \mathrm{g} / 100 \mathrm{~g})$, podendo ser utilizado no combate à hipovitaminose A. A irradiação dos frutos de buriti na dose de 0,5 kGy não alterou significativamente os teores de carotenóides e açúcares. Entretanto, houve uma redução na concentração do ácido ascórbico com o aumento da dose, que pode ter sido causada pela irradiação ou por fatores intrínsecos e extrínsecos que alteram a estabilidade do ácido ascórbico nos alimentos, podendo converter o ácido ascórbico a dehidroascórbico, mantendo ainda a forma ativa da vitamina C.

PALAVRaS-chave: Buriti, Irradiação Gama, Carotenóides, CLAE, Ácido Ascórbico, Açúcares.

\section{Evaluation of Gamma Irradiation Effects on Carotenoids, Ascorbic Acid and Sugar Contents of Buriti Fruit (Mauritia flexuosa L.)}

\begin{abstract}
Buriti (Mauritia flexuosa L.), a typical fruit from the Northeast and Center-West Amazon of Brazil, is used in many regional dishes. It is considered an excellent source of carotenoids that are A vitamin precursors, showing a majority of $\beta$-carotene. It also presents ascorbic acid and sugar contents. Many studies have indicated that the lack of A vitamin is the main cause of night blindness and xerophthalmia. Also, ascorbic acid deficiency may cause scorbutic disease. The use of food irradiation is growing and represents an economic benefit to agriculture through the reduction of post-harvesting losses while maintaining food nutritional quality. In this study, Buriti in natura was treated with gamma irradiation with doses of $0.5 \mathrm{kGy}$ and $1.0 \mathrm{kGy}$. The objective was to evaluate the irradiation effects on total carotenoids, ascorbic acid and sugars concentrations of Buriti. The fruit was evaluated through the total carotenoids analysis, by spectrophotometry, and the carotenoids ( $\alpha$ and $\beta$-carotene and lutein), ascorbic acid and sugars were determined by High Performance Liquid Chromatography (HPLC). The results showed that buriti is an excellent source of total carotenoids $(44600 \mu \mathrm{g} / 100 \mathrm{~g})$. The irradiation of buriti with the dose of $0.5 \mathrm{kGy}$ did not significantly change carotenoids and sugars contents. However, there was a reduction of ascorbic acid concentration with an increase of the dose, which may have been caused by irradiation or by intrinsic and extrinsic factors that alter ascorbic acid stability in food, converting ascorbic to dehydroascorbic acid, while keeping the $\mathrm{C}$ vitamin active form.
\end{abstract}

KEY WORDS: Buriti, Gamma Irradiation, Carotenoids, HPLC, Ascorbic Acid, Sugars.

\footnotetext{
1 Instituto Militar de Engenharia, Seção de Engenharia Química, Química Orgânica e de Produtos Naturais. Avenida Marechal Fontenelle, 4784, casa 06, Vila City, R 3, Jardim Sulacap CEP 21750-001. e-mail: santoslima@ime.eb.br

2 Instituto Militar de Engenharia, Seção de Engenharia Nuclear, Ciência e Tecnologia de Alimentos, Irradiação de Alimentos. E-mail: keila@ime.eb.br

${ }^{3}$ Instituto Militar de Engenharia, Seção de Engenharia Nuclear, Engenharia Ambiental. E-mail: maysa@ime.eb.br

${ }^{4}$ Instituto Militar de Engenharia, Seção de Engenharia Nuclear, Irradiação de Alimentos. E-mail: jaquelinefisica@bol.com.br

${ }^{5}$ Embrapa Agroindústria de Alimentos, Laboratório de Cromatografia Líquida, Química Orgânica e de Produtos Naturais. E-mail: ronoel@ctaa.embrapa.br

${ }^{6}$ Embrapa Agroindústria de Alimentos, Laboratório de Cromatografia Líquida, Ciência e Tecnologia de Alimentos. E-mail: sidney@ctaa.embrapa.br
} 


\section{INTRODUÇÃO}

Alimentos de origem vegetal, como as hortaliças e frutas, desempenham um importante papel na alimentação humana devido ao valor nutricional e atributos sensoriais (Lima et al., 2004). Levando em consideração a necessidade e a importância da nutrição para os seres humanos e a perecibilidade de hortaliças e frutas, faz-se necessário o estudo da aplicação de diferentes métodos de conservação como o emprego do calor, do frio, da salga, de aditivos químicos, da radiação e outros (Gould, 1996).

Dentre todos os métodos de conservação de alimentos destacamos a radiação. O mérito da irradiação de alimentos está em sua capacidade de destruir microorganismos patogênicos e deteriorantes presentes nos alimentos sem alterar, dependendo da dose, seu sabor e sua qualidade nutricional. É, empregada, ainda, para eliminar insetos e retardar o processo germinativo em alimentos de origem vegetal. Desta forma, há um aumento na segurança dos alimentos destinados ao consumo humano e uma redução nas perdas causadas por deterioração, além de aumentar do tempo de vida útil (Hagenmaier \& Baker, 1998).

Estudos realizados pelo Comitê de especialistas da Food and Agriculture Organization (FAO), World Health Organization (WHO) e International Atomic Energy Agency (IAEA), mostraram que qualquer alimento irradiado na dose média de 10 kGy é seguro e não induz problemas nutricionais, além de eliminar microrganismos contaminantes e prejudiciais ao homem (Lima et al., 2001). Entretanto, em 1997 o comitê FAO/ IAEA/ WHO se reuniu e concluiu que a irradiação em altas doses é semelhante ao convencional processamento térmico, como a esterilização de alimentos de baixa acidez, em que se elimina o perigo biológico dos alimentos destinados ao consumo humano, sem acarretar alteraçôes físicas ou químicas que se constituam em um perigo (Diehl, 2002).

O Buriti (Mauritia flexuosa L.), fruta nativa da Amazônia, Nordeste, Centro Oeste e Brasil Central, atingindo o norte do estado de São Paulo, é uma drupa globoso-alongada de 4-7 cm de comprimento constituída de epicarpo formado de escamas rombóides de cor castanho-avermelhada, mesocarpo representado por uma massa espessa de cor alaranjada e endocarpo esponjoso que envolve a semente muito dura. Esta fruta de origem nativa é uma fonte de alimento para as populaçóes de baixa renda, pois possui adequados valores nutricionais, destacando-se os carotenóides e o ácido ascórbico (Lorenzi et al., 2006; EMBRAPA, 2007; Silva et al., 2007).

Os carotenóides compóem um dos grupos de pigmentos naturais mais extensamente encontrados na natureza, responsáveis pelas colorações do amarelo ao vermelho de flores, folhas, frutas, algumas raízes (cenoura), gema de ovo, lagosta e outros crustáceos, peixes e aves (Lima et al., 2004; Britton, 1992). Além de serem corantes naturais dos alimentos, os carotenóides apresentam efeitos benéficos à saúde humana como atividade de provitamina $\mathrm{A}$, aumento da resposta imune e redução do risco de doenças degenerativas como câncer, degeneração macular, catarata e doenças cardiovasculares (Sentanin \& Rodriguez-Amaya, 2007).

O ácido ascórbico é conhecido como promotor de numerosos processos químicos, bioquímicos e fisiológicos, tanto em animais como em plantas. Desempenha várias funções no organismo relacionadas ao sistema imune, formação de colágeno, absorção de ferro, inibição da formação de nitrosaminas e atividade antioxidante. $\mathrm{O}$ seu conteúdo pode ser influenciado pelo tipo de solo, forma de cultivo, condições climáticas, procedimentos agrícolas para a colheita e armazenamento. Além disso, o ácido ascórbico, em sua forma pura, é bastante instável, sendo facilmente destruídos por oxidação, particularmente temperatura elevadas, luz, umidade, alcalinidade, catalisadores metálicos e danos físicos (O'Keefe, 2001; Silva et al., 2004).

Os açúcares, como a sacarose, frutose e a glicose, juntamente com os polissacarídeos, como o amido e a celulose, são os principais componentes do grupo de substâncias chamadas de carboidratos ou compostos poliidroxílicos alifáticos. A fórmula empírica da grande maioria dos carboidratos encontrada em alimentos corresponde a $\left(\mathrm{CH}_{2} \mathrm{O}\right)$ n' de onde surgiu o seu nome. As moléculas de glicose podem se combinar e formar outros componentes característicos das plantas como por exemplo a sacarose (ligaçôes glicosídicas $\alpha$ $1-4$ ), a celulose (ligações glicosídicas $\beta 1-4$, em cadeia não ramificada) e o amido (ligações glicosídicas $\alpha$ 1-4 e $\alpha$ 1-6 nos pontos de ramificação) (Coultate, 2004).

O objetivo deste trabalho é analisar os efeitos da radiação gama nas doses de 0,5 kGy e 1,0 kGy, a partir de fonte de Césio 137, na concentração de ácido ascórbico, carotenóides totais, luteína, $\alpha$ e $\beta$-carotenos e açúcares no Buriti.

\section{MATERIAIS E MÉTODOS}

O Buriti (Mauritia flexuosa L.) analisado foi adquirido na cidade de Abadia de Goiás, Goiás (GO), no mês de fevereiro de 2007 no estádio de maturação pronto para consumo. Por transporte aéreo foi encaminhado para a Seção de Engenharia Nuclear, do Instituto Militar de Engenharia (IME), localizado na cidade do Rio de Janeiro. Os frutos foram retirados da embalagem de remessa onde foram selecionados, classificados, pesados e sanitizados em água clorada na proporção 5-10 $\mathrm{L}$ de água por $1 \mathrm{~kg}$ de fruto, contendo $150 \mathrm{mg} / \mathrm{L}$ de cloro. Os buritis considerados como próprios para o consumo foram então imediatamente acondicionados em embalagens flexíveis de polietileno (PE), adequadamente identificados e armazenados sob congelamento $\left(-18^{\circ} \mathrm{C}\right)$, para resguardálos de qualquer alteração, por se tratar de amostra sujeita à deterioração. $\mathrm{O}$ armazenamento em temperaturas de 
congelamento e refrigeração contribuem para a redução das atividades microbianas e das alteraçôes químicas e enzimáticas características das hortaliças e frutas. Na véspera da realização das análises, os frutos acondicionados e armazenados sob congelamento foram transferidos para o refrigerador $\left(7-10^{\circ} \mathrm{C}\right)$ com a finalidade de ocorrer o descongelamento por um período de 12 a 16 horas.

Os frutos foram descascados manualmente com facas de aço inoxidável sanitizadas. A extração da polpa do buriti também foi realizada manualmente. A amostra para análise foi oriunda da mistura e homogeneização da polpa de 6-8 unidades de frutos. Uma análise da quantidade de carotenóides totais, luteína, $\alpha$ e $\beta$-carotenos do Buriti foi realizada em uma amostra do lote, com 1,0128 g de polpa, para o tempo " $\mathrm{t}$ " (logo após o recebimento dos frutos).

Em seguida os frutos foram encaminhados para serem irradiados, com as doses de 0,5 e 1,0 kGy, em um irradiador de pesquisa localizado no Centro Tecnológico de Exército CTEx, Rio de Janeiro, sendo o tempo de exposição calculado considerando-se a atividade atual da fonte, a dose média desejada (Gy), o diâmetro ou altura máxima da amostra, densidade e geometria da amostra, a alta-atenuação e o fator de Build-up. $\mathrm{O}$ irradiador utilizado tem capacidade de prover simultaneidade a duas câmaras com volume total de 80 litros, uma taxa de dose máxima igual a $2 \mathrm{kGy} / \mathrm{h}$ e com boa uniformidade, sendo a razão entre dose máxima e mínima inferior a $20 \%$. O irradiador é do tipo cavidade com fonte de césio 137 autorecolhível, controlada por um sistema eletro-eletrônico, com portas blindadas móveis. $\mathrm{O}$ irradiador possui ainda vários sistemas de segurança, os quais incluem dispositivos lógicos e travas fixas, que garantem o funcionamento seguro, eliminando qualquer possibilidade de exposição acidental à fonte (Lima et al., 2001).

Após a irradiação os frutos (controle e os irradiados) foram transportados para a Embrapa Agroindústria de Alimentos, Rio de Janeiro, aonde os carotenóides totais foram obtidos por espectrofotometria e os carotenos ( $\alpha$ e $\beta$-caroteno) e luteína por Cromatografia líquida de Alta Eficiência - CLAE, para um tempo $t_{1}$ (um mês sob congelamento). Os carotenóides foram extraídos com acetona e em seguida sofreram partição para éter de petróleo, sendo concentrados, através da evaporação do solvente sob fluxo de nitrogênio, e diluídos em acetona $1 \mu \mathrm{L}$ (Lima et al., 2004; Magalhães et al., 2007). A fase móvel foi transferida para balão volumétrico $(100 \mathrm{ml})$ através de funil com sulfato de sódio anidro e quantificados por espectrofotometria a $450 \mathrm{~nm}$. A análise cromatográfica foi realizada num cromatógrafo da Shimadzu utilizando coluna cromatográfica C30 3um 4.6 x 250mm - YMC Carotenoid WATERS, sendo a fase móvel o gradiente de Metanol/Metil t-Butil Éter - 80:20 para 10:90 em 28 minutos, com fluxo $0,8 \mathrm{~mL} / \mathrm{min}$ e temperatura de $30^{\circ} \mathrm{C}$.
A análise das concentrações do ácido ascórbico foi obtida através da CLAE, utilizando-se o mesmo cromatógrafo e o mesmo tempo $\mathrm{t}_{1}$, aonde uma solução $0,5 \mathrm{M}$ de ácido sulfúrico concentrado supra puro foi usada para extração, padrão de ácido L-ascórbico, coluna de troca iônica forma ácida BIO RAD HPX 87H (7,8 x $300 \mathrm{~mm})$, detector UV 243,8 nm, 10 min para o tempo de corrida, $20 \mu \mathrm{L}$ de volume de injeção, $5^{\circ} \mathrm{C}$ de temperatura do injetor, vazão de fase móvel igual a $0,7 \mathrm{~mL} / \mathrm{min}$, pressão máxima de 1500 psi e ácido sulfúrico 0,1 M como fase móvel.

As análises dos açúcares também foram realizadas utilizando a cromatografia líquida de alta eficiência - CLAE, com o cromatógrafo da Shimadzu para o mesmo tempo $t_{1}$, com as seguintes características: a) fase móvel - acetonitrila $75 \%$; b) detector - IR 2410; c) fluxo - 1,4 ml/min e d) coluna - Amino $30 \mathrm{~cm}$ x 4,6 mm.

Todas as amostras da polpa de buriti controle e irradiados nas diferentes doses foram analisadas em triplicata para determinação de carotenóides, ácido ascórbico e açúcares.

Os resultados obtidos das determinações químicas do buriti foram submetidos à análise de variância (ANOVA) e as médias dos tratamentos foram comparados pelo teste $t$-Student para um nível de significância de 5\% (Zar, 1996).

\section{RESULTADOS E DISCUSSÃO}

Para o tempo $\mathrm{t}_{0}$ obteve-se $44600 \mu \mathrm{g} / 100 \mathrm{~g}$ de carotenóides totais, sendo $70 \%$ de $\beta$-caroteno, $12 \%$ de $\alpha$-caroteno, e 1,6 $\%$ de luteína. A Figura 1 mostra um cromatograma típico dos carotenóides presentes no Buriti.

Neste cromatograma observa-se a presença de quatro picos maiores, sendo os carotenóides identificados de acordo com a ordem de eluição na coluna cromatográfica, respectivamente, 9-cis- $\beta$-caroteno, $\alpha$-caroteno, $\beta$-caroteno e 13 -cis- $\beta$ caroteno. Os espectros de absorção, região do UV-Visível, dos carotenóides estudados, comprimento de onda de 450 nm, estão apresentados na Figura 2. O emprego do detector por conjunto de diodos permitiu a obtenção de espectros de absorção que auxiliaram na verificação da pureza dos picos,

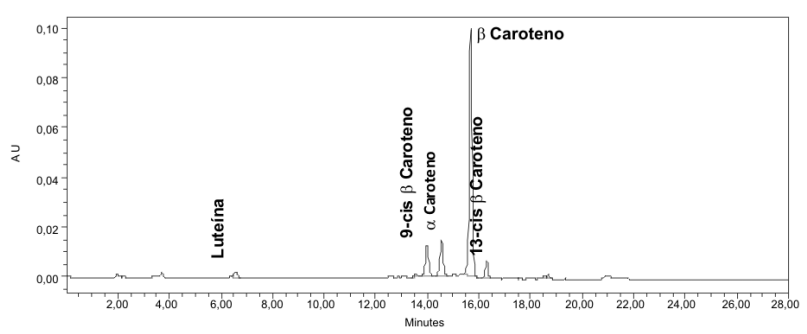

Figura 1 - Cromatograma dos carotenóides do Buriti (Mauritia flexuosa L.) para 0 tempo $t_{0}$, com o tempo de retenção de $\alpha$ e $\beta$-caroteno e luteína. 
Tabela 1 - Quantidade de carotenóides ${ }^{1}$, ácido ascórbicoe e açúcares ${ }^{1}$ em Buritis não irradiados (controle) e irradiados com doses de 0,5 kGy e 1,0 kGy.

\begin{tabular}{lcccccc}
\hline $\begin{array}{l}\text { Controle e Doses } \\
(\mathrm{kGy})\end{array}$ & $\begin{array}{c}\text { Carotenóides Totais } \\
(\mu \mathrm{g} / 100 \mathrm{~g})\end{array}$ & $\begin{array}{c}\alpha \text {-Caroteno } \\
(\mu \mathrm{g} / 100 \mathrm{~g})\end{array}$ & $\begin{array}{c}\beta \text {-Caroteno } \\
(\mu \mathrm{g} / 100 \mathrm{~g})\end{array}$ & $\begin{array}{c}\text { Concentração de ácido } \\
\text { ascórbico }(\mathrm{mg} / 100 \mathrm{~g})\end{array}$ & $\begin{array}{c}\text { Frutose } \\
(\mathrm{mg} / 100 \mathrm{~g})\end{array}$ & $\begin{array}{c}\text { Glicose } \\
(\mathrm{mg} / 100 \mathrm{~g})\end{array}$ \\
\hline Controle & $37211^{\mathrm{a}}$ & $3625^{\mathrm{a}}$ & $34085^{\mathrm{a}}$ & $31,86^{\mathrm{a}}$ & $0,43^{\mathrm{a}}$ & $0,15^{\mathrm{a}}$ \\
0,5 & $38620^{\mathrm{a}}$ & $2924^{\mathrm{b}}$ & $36667^{\mathrm{a}}$ & $23,33^{\mathrm{b}}$ & $0,55^{\mathrm{a}}$ & $0,16^{\mathrm{a}}$ \\
1,0 & $22720^{\mathrm{b}}$ & $4288^{\mathrm{b}}$ & $18048^{\mathrm{b}}$ & $23,74^{\mathrm{b}}$ & $0,28^{\mathrm{b}}$ & $0,08^{\mathrm{b}}$ \\
\hline
\end{tabular}

1. Valores médios com letras diferentes na mesma coluna indicam diferença significativa $(p<0,05)$.

bem como na separação dos isômeros cis e trans do $\beta$-caroteno (Kimura \& Rodriguez-Amaya, 2003).

Os resultados de carotenóides totais, $\alpha$ e $\beta$-caroteno, ácido ascórbico e açúcares para as doses de 0,5 kGy e 1,0 kGy, obtidas em amostras de Buritis irradiados e no controle, para o tempo $t_{1}$, estão representados na Tabela 1 .

Os Buritis não irradiados apresentaram teores de carotenóides entre 37211 e $44600 \mu \mathrm{g} / 100 \mathrm{~g}$, mostrando que são excelentes fontes de provitamina $\mathrm{A}$, estando acima dos teores normalmente encontrados em cenouras consideradas a fonte mais conhecida e aceita pelos consumidores (Lima et al., 2004).

Houve um decréscimo de aproximadamente 17\% no teor de carotenóides totais nos Buritis não irradiados entre a análise $\mathrm{t}_{0}(44600 \mu \mathrm{g} / 100 \mathrm{~g})$ e $\mathrm{t}_{1}(37211 \mu \mathrm{g} / / 100 \mathrm{~g})$. O tempo de armazenamento de 1 mês e o congelamento são as possíveis causas desta redução.

Os resultados das amostras irradiadas mostram que na dose de 1,0 kGy houve uma redução significativa do teor de carotenóides totais para um nível de significância de 5\% (Teste t-Student). LIMA et al. (2004), em estudos com cenouras irradiadas, também observaram a redução dos carotenóides majoritários com o aumento de dose de radiação e propuseram uma rota de degradação dos carotenóides através de oxidação e quebra das ligações químicas em e iononas, compostos conhecidos atualmente por químicos e aromistas como responsáveis pelo aroma em vegetais. A degradação dos carotenóides totais e a auto-oxidação dos ácidos graxos insaturados nos alimentos podem ocorrer devido a oxidação direta ou por efeitos indiretos de radicais livres. Na oxidação ocorre o ataque do oxigênio singleto ${ }^{1} \mathrm{O}_{2}$, oxigênio de alta energia, ao carbono adjacente a dupla ligação, do grupo -metileno $\left(\mathrm{CH}_{2}\right)$, ou diretamente reage com a dupla ligação, resultando na formação de hidroperóxidos. Os radicais livres são moléculas que possuem vida muito curta e são altamente reativos, enquanto buscam um aceptor para seu elétron livre não-pareado. A energia das radiaçôes, os metais, o oxigênio, a temperatura e a luz são conhecidos como catalisadores das reaçôes de degradação dos carotenóides e dos ácidos graxos insaturados nos alimentos (Coultate, 2004; Uenojo et al., 2007).

O teor de ácido ascórbico, $31,86 \mathrm{mg} / 100 \mathrm{~g}$, encontrado na amostra controle de Buriti mostrou uma redução significativa de $27 \%$ e $25 \%$ nas doses, respectivamente, de $0,5 \mathrm{kGy}$ e 1,0 kGy. Os resultados foram semelhantes ao de JUSTI et al. (2000) que encontraram redução de $23 \%$ no conteúdo de vitamina $\mathrm{C}$ na polpa de camu-camu, armazenada a $-18^{\circ} \mathrm{C}$ durante 28 dias. Os autores também verificaram diferentes valores de ácido ascórbico no camu-camu conforme a região do Brasil. Frutas colhidas no Estado do Paraná, região Sul, apresentaram redução de 47 a 58\% de ácido ascórbico, em
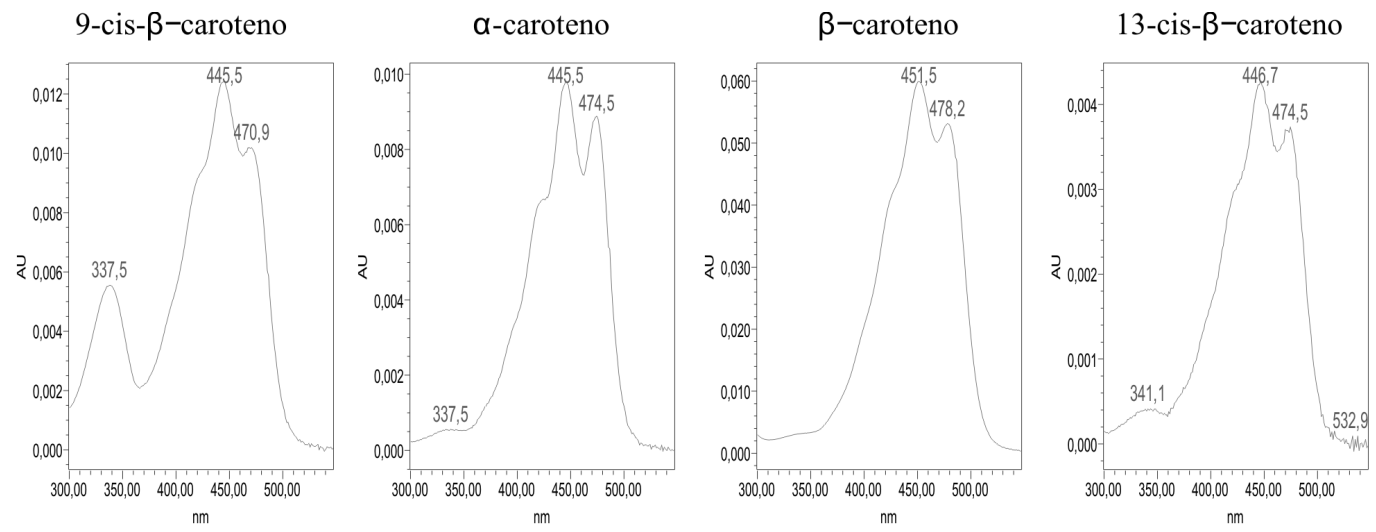

Figura 2 - Espectros do 9-cis- $\beta$-caroteno, $\alpha$-caroteno, $\beta$-caroteno e 13-cis- $\beta$-caroteno, para um comprimento de onda igual a $450 \mathrm{~nm}$, do Buriti (Mauritia flexuosa L.). 
relação aos teores de frutas colhidas no Amazonas, região Norte, podendo ser causado por variaçóes climáticas e características do solo. Segundo Silva et al. (2004) a concentração de vitamina C na amostra pode variar por influência da temperatura, do oxigênio, do $\mathrm{pH}$, do tempo de congelamento, da variação natural da composição de frutos nativos, bem como da facilidade de oxidação.

Nas análises da polpa do buriti controle e irradiado, não foi encontrada nenhuma concentração do dissacarídeo sacarose, mas foram identificadas através da CLAE quantidades dos monossacarídeos frutose e glicose. As concentraçōes de açúcares quantificadas estão na Tabela 1 . Os dados obtidos mostraram, através do teste estatístico t-Student, que não há influência da dose na concentração dos açúcares para a dose de $0,5 \mathrm{kGy}$, mas houve redução significativa dos açúcares na dose de 1,0 kGy com um nível de significância de 5\%.

A ausência de sacarose pode ser justificada como conseqüência da quebra das ligações glicosídicas, resultando nos monossacarídeos glicose e frutose, devido ao avançado estádio de maturação do fruto, o armazenamento congelado, a possível conversão em ácidos graxos ou como substrato energético para o metabolismo de microrganismos. Este fato foi observado tanto no buriti controle como nos irradiados.

\section{CONCLUSÃO}

As análises utilizando espectrofotometria e CLAE indicaram que o Buriti é uma excelente fonte de carotenóides totais e $\beta$-caroteno, podendo ser empregado no combate a hipovitaminose A com alta prevalência na população brasileira.

A dose de 1,0 kGy causou redução na concentração dos carotenóides totais do Buriti, contudo continua sendo uma fonte excepcional de pró-vitamina $\mathrm{A}$, pois $100 \mathrm{~g}$ de polpa pode contribuir com aproximadamente três vezes as necessidades diárias de vitamina $\mathrm{A}$, que é de $900 \mu \mathrm{g}$, para um homem acima de 14 anos, conforme o Instituto Americano de Medicina (IOM, 2001). Além disso, a diminuição do teor de carotenóides pelo armazenamento congelado teve efeito semelhante ao da irradiação.

Os resultados do ácido ascórbico por CLAE para os Buritis irradiados mostram que houve redução da concentração com o aumento da dose.

A concentração dos açúcares do buriti foi mantida após o emprego da dose de $0,5 \mathrm{kGy}$. Contudo, houve redução significativa dos açúcares na dose de $1 \mathrm{kGy}$ para um nível de significância de 5\%.

Os dados obtidos indicam a radiação como um método de grande potencial para conservação de alimento, uma vez que as alteraçôes químicas não diferem das observadas em outros métodos quanto as modificações do valor nutricional do Buriti do Brejo (Mauritia flexuosa L.).

\section{AGRADECIMENTOS}

Os autores agradecem a Embrapa Agroindústria de Alimentos, ao Centro Tecnológico do Exército, ao Centro Regional de Ciências Radiológicas - Goiânia (CRCN/CNEN) e a Coordenação de Aperfeiçoamento de Pessoal de Nível Superior (CAPES).

\section{BIBLIOGRAFIA CITADA}

Britton, G. 1992. Carotenoids. In: Hendry, G.F. Natural foods colorants. New York: Blackie (Eds), p. 141-148.

Coultate, T. P. 2004. Alimentos. A Quimica de seus Componentes, Terceira Edição. Porto Alegre, Artmed Editora S.A.

Diehl, J.F. 2002. Food Irradiation-Past, Present and Future. Radiation Physics and Chemistry, 63(1): 211-215.

EMBRAPA, 2007. Frutas Nativas do Cerrado. Embrapa cerrado. Disponível em http://www.cpac.embrapa.br/tecnologia/ floranativa.html. Acesso em 0/03/2007.

Gould, G.W. 1996. Methods for Preservation and Extension of Shelf Life. International Journal of Food Microbiology, 33: 51-64.

Hagenmaier, R.D.; Baker, R.A 1998. Microbial Population of Shredded Carrot in Modified Atmosphere Packaging as Related to Irradiation Treatment. Journal of Food Science, 63(1): 162164.

IOM, 2001. Dietary Reference Intake: for Vitamin A, Vitamin K, Arsenic, Boron, Cromium, Copper, Iodine, Iron, Manganese, Molybdenium, Nickel, Silicon, anadium and Zinc. Disponível em http://www.iom.edu/?id=21381. Acesso em 18/04/2007.

Justi, K.C.; Visentainer, J.V.; Souza, N.E.; Matsushita, M. 2000. Nutritional Composition and Vitamin C Stability in Stored Camu-camu (Myrciaria dúbia) pulp. Archivos Latinoamericanos de Nutricion, 50(4): 405-408.

Kimura, M.; Rodriguez-Amaya, D.B. 2003. Estabelecimento das Condiçôes Cromatográficas para Análise de Carotenóides em Alimentos por CLAE: uma Ciência ou uma Arte. Boletim da Sociedade Brasileira de Ciência e Tecnologia de Alimentos, 37(2): 74-81.

Lima, K.S.C.; Grossi, J.L.S.; Lima, A.L.S.; Alves, P.F.M.P.; Coneglian, R.C.C.; Godoy, R.L.O.; Sabaa-Srur, A.U. 2001. Efeitos da Irradiação Ionizante na Qualidade Pós-colheita de Cenouras (Daucus carota L.) cv. Nantes. Ciência e Tecnologia de Alimentos, 21(2): 202-208

Lima, K.S.C.; Lima, A.L.S.; Freitas, L.C.; Della-Modesta, R.C.; Godoy, R.L. 2004. O Efeito de Baixas Doses de Irradiação nos Carotenóides Majoritários em Cenouras Prontas para o Consumo. Ciência e Tecnologia de Alimentos, 24(2): 183-193.

Lorenzi, H.; Bacher, L.B.; Lacerda, M.T.C.; Sartori, S.F. 2006. Frutas Brasileiras e Exóticas Cultivadas. São Paulo, Instituto Plantarum de Estudos da Flora Ltda. 
Magalhães, L.A.M.; Lima, M.P.; Marinho, H.A.; Ferreira, A.G. 2007. Identificação de Bergenina e Carotenóides no Fruto de Uchi (Endopleura uchi, Humiriaceae). Acta Amazonica, 37(3): 447-450.

O'Keefe, T. 2001. Ascorbic Acid and Stable Ascorbate Esters as Sources of Vitamin C in Aquaculture Feeds. Singapore: American Soybean Association - United Soybean Board, 8 p. (ASA Technical Bulletin Vol. AQ48-2001).

Silva, J.M.; Coelho, M.J.; Lima, K.S.C.; Lima, A.L.S.; Godoy, R.L.O.; Pacheco, S. e Ferreira, R.S. 2007. Evaluation of Carotenoid Contents in Irradiated Buriti (Mauritia flexuosa L.). In: 2007 International Nuclear Atlantic Conference. SantosSP. $5 \mathrm{pp}$.

Silva, M.R.; Silva, M.S.; Oliveira, J.S. 2004. Estabilidade de Ácido Ascórbico em Pseudofrutos de Caju-do-Cerrado Refrigerados e Congelados. Pesquisa Agropecuária Tropical, 34(1): 9-14.
Sentanin, M.A.; Rodriguez-Amaya, D.B. 2007. Teores de Carotenóides em Mamão e Pêssego Determinados por Cromatografia Líquida de Alta Eficiência. Ciência e Tecnologia de Alimentos, 27(1): 13-19.

Uenojo, M.; Maróstica Júnior, M. R.; Pastore, G. M. 2007. Carotenóides: Propriedades, Aplicações e Biotransformação para Formação de Compostos de Aroma. Química Nova, 30 (3): 616-622

Zar, J.H. 1996. Biostatistical Analysis, Third Edition. New Jersey: Prentice Hall.

Recebido em 31/07/2008

Aceito em 01/12/2008 